Indonesian Journal of Cardiology

Indonesian J Cardiol 202 I:42:76-80

pISSN: 0I26-3773 / elSSN: 2620-4762 doi: $|0.3070| / j j c .|| 3 \mid$

\title{
Patent Foramen Ovale Closure Procedure
}

\author{
Doni Firman, Arwin Saleh Mangkuanom
}

Departement of Cardiology and Vascular Medicine, Universitas Indonesia / National Cardiovascular Center Harapan Kita, Jakarta Indonesia.

\section{Correspondence:}

Doni Firman

Departement of Cardiology and Vascular Medicine, Universitas Indonesia / National Cardiovascular Center Harapan Kita, Jakarta Indonesia.

Email: dr.donifirman@gmail.com

\begin{abstract}
Patent foramen ovale is strongly associated with cryptogenic stroke. Various clinical trials has shown the association between cryptogenic stroke and incidence of undelrying patent foramen ovale, these trials also shown the decrease of cryptogenic stroke incidence with the treatment of patent foramen ovale Lesion. In the absence of absolute contraindications, patients with patent foramen ovale are advised to undergo closure. Preprocedural examinations such as trans esophageal echocardiography and pretreatment with anticoagulants are required to prevent peri and postprocedural adverse events. Currently, patent foramen ovale Closure can be done through a percutaneous access with minimal risk. Treatment of patent foramen ovale can help decrease future incidences of strokes
\end{abstract}

(Indonesian J Cardiol. 2021;42:76-80)

Keywords: structural; interventional; patent foramen ovale; percutaneous; stroke 


\section{Introduction}

$\mathrm{P}$ atent foramen ovale is being thought to be one of the factors causing cryptogenic stroke and about $40 \%$ patient cryptogenic stroke has patent foramen ovale. ${ }^{1}$ It takes centuries \& there are numerous lessons to be learned from the patent foramen ovale history until an effective treatment. In 1490, DaVinci first describe communication between the atria in pigs and ox. Virchow in 1570, first mentioned about physiologic importance of the structure in the fetal circulation $\&$ the phenomenon of embolization in the vascular system. Julius Cohnheim one of Virchow's students, in 1877 described a paradoxical embolism through the patent foramen ovale. Starting in the 1900s the closure of patent foramen ovale was considered as one of the effective therapies. Blakemore in 1939 \& Murray in 1948 did the first surgical patent foramen ovale closures. Finally, in 1992, the first percutaneous patent foramen ovale closures were conducted by Lockin 36 patients with known right-to-left atrial shunting and presumed paradoxical emboli. ${ }^{2,3}$

\section{Previous Studies on Patent Foramen Ovale Closure}

Since then a lot of devices have been made $\&$ design specially for patent foramen ovale closure from many factories, but unfortunately, not all of them could perform equally well. As an example the STARFlex Septal Closure System (NMT Medical Inc., Boston) of the CLOSURE I trial, 2012, demonstrated lower implantation success and closure rates $89 \%$ and $86 \%$, respectively) than other patent foramen ovale occlusion devices at that time, on other hand, it also showed no significant advantage of patent foramen ovale closure makes dampened the enthusiasm tremendously for doing this procedure. The Incidence of postimplantation atrial fibrillation and atrial thrombus formation at 6 -month rates was relatively high $(5.7 \%$ and $1.1 \%$, respectively) that cause stroke in half of the patients who have thrombus.

Long-term follow-up trials examining the effectiveness of devices for the prevention of an event, the risk of which is low at baseline and cumulative over time was well demonstrated by the RESPECT (Randomized Evaluation of Recurrent Stroke Comparing patent foramen ovale Closure to Established
Current Standard of Care) trial which randomized 980 stroke patients to patent foramen ovale closure versus medical management. ${ }^{4}$ In the short-term analysis (2.6year median follow-up), there was a strong trend toward a benefit for patent foramen ovale closure but did not reach statistical significance $(\mathrm{P}=0.08)$. However, after a long-term follow-up of 5.9 years, RESPECT showed a more pronounced, now statistically significant stroke risk reduction with patent foramen ovale closure compared to medical therapy.

Appropriate patient selection is another important lesson, these kinds of criteria have been described well from several trials, and all of them showing a benefit of patent foramen ovale closure compared to medical therapy alone. In the RESPECT trial, only patients with patent foramen ovale who had objective evidence of a stroke by neuroimaging were enrolled. ${ }^{5}$ CLOSE (patent foramen ovale Closure or Anticoagulation vs. Antiplatelets after Stroke) trial, including only patients with an atrial septal aneurysm or large shunt. ${ }^{6}$ DEFENSE-patent foramen ovale (Cryptogenic Stroke and High-Risk patent foramen ovale) trials conducted in Korea, having only those with a hypermobile interatrial septum, atrial septal aneurysm, or a separation of the septum primum from secundum of $12 \mathrm{~mm}$ as an inclusion criterion. ${ }^{7}$ REDUCE (patent foramen ovale Closure or Antiplatelet Therapy for Cryptogenic Stroke) trial, exclude patients with evidence for small vessel ischemic disease, i.e., prior lacunar infarct(s), uncontrolled diabetes mellitus or hypertension, autoimmune disease, and alcohol abuse. ${ }^{8}$

\section{Indications and Contraindications for Patent Foramen Ovale Closure}

In real-world clinical practice, it is not that simple to prove cryptogenic stroke related to patent foramen ovale and then to make decisions for patent foramen ovale closure. The RoPE score helps us to calculate the possibility of cryptogenic stroke caused by patent foramen ovale, it is also can be used to predict the risk of recurrent stroke and mortality risk after the patent foramen ovale closure procedure. RoPE score of $\leq 6$ indicates a high risk of recurrent paradoxical embolism and mortality after patent foramen ovale closure. ${ }^{9}$

Meta-analysis of Randomized Controlled Trials (RCT) has shown the benefit of patent foramen ovale closure to reduce the risk of recurrent stroke in high- 


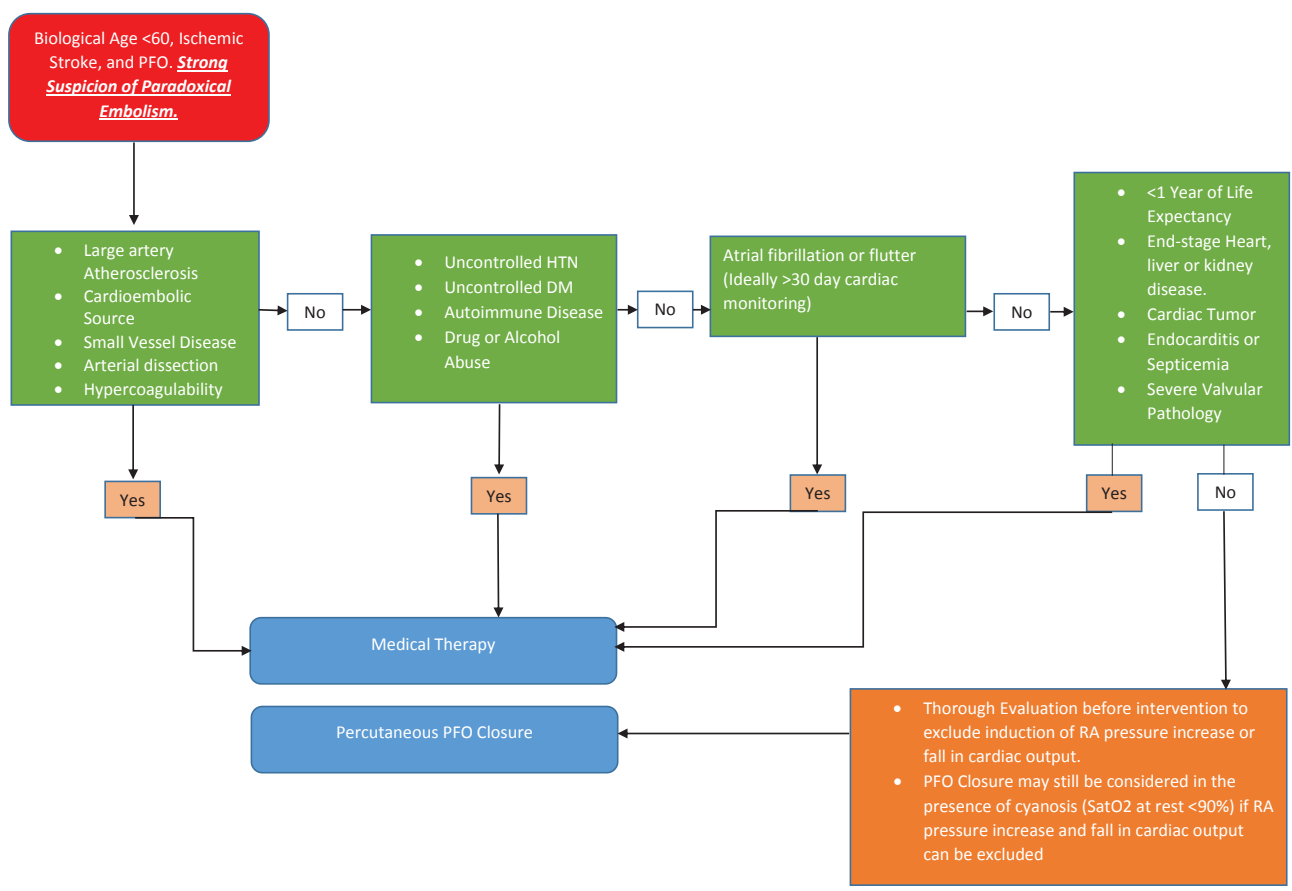

Figure 1. Evidence-Based Algorithm for patent foramen ovale Closure in Ischemic Stroke Patients for Highest Clinical Yield, Based on Randomized Trials and Guidelines. (Adapted from Mojadidi et al Cryptogenic Stroke and patent foramen ovale. J Am Coll Cardiol 2018;71(9):1035-1043 and Baumgartner et al, 2020 ESC Guidelines for the management of adult congenital heart disease. Eur Heart J. 2021;42(6):563-645. ). ${ }^{13,14}$

risk patient criteria. ${ }^{10}$ According to a meta-analysis of absolute mean reduction in the risk of recurrent stroke at patent foramen ovale closure is only 1.0 per 100 patients per year, however, long-term prevention of secondary stroke prevention in young patients should be considered. ${ }^{11}$ In RESPECT, CLOSE dan REDUCE trial, participants who enrolled were categorized as a young adult below 60 years old with an average of 51.2 years old in DEFENSE trial. ${ }^{5,6,8,7}$ Other meta-analyses showed that patent foramen ovale closure increases the incidence of new-onset atrial fibrillation and atrial flutter compared to medical treatment only. ${ }^{12}$

When patients came with a cryptogenic stroke, age under 60 years old $\&$ have an indication for patent foramen ovale closure, it is recommended to have a team who systematically review \& make a decision for the patent foramen ovale closure procedure as seen in picture 1 , which consist of multidisciplinary specialist doctors.

Patent foramen ovale closure is recommended in patients with cryptogenic stroke who have been well investigated systematically and have high-risk morphology of patent foramen ovale. Patent foramen ovale closure is not recommended in pregnant cryptogenic stroke patient and one who cannot receive antiplatelet treatment after the procedure.

\section{Patent Foramen Ovale Closure Procedure}

Percutaneous patent foramen ovale Closure can be performed in a standard catheterization laboratory under fluoroscopic and Transesophageal Echocardiography (TEE) guidance. General anesthesia is almost required to facilitate TEE. Adequate anticoagulation using unfractionated heparin $80-100 \mathrm{IU} / \mathrm{kg}$ body weight, administered intravenously. The femoral vein is used as a puncture site, crossing the patent foramen ovale channel using Multipurpose (MP) Catheter 6F with guidewire $\&$ directly to the left upper pulmonary vein. Patent foramen ovale lesion sizing can be done using 
a balloon with angiographic analysis or by TEE image which can obtain information more accurately. A left anterior oblique fluoroscopy projection will show a good septum profile.

After sizing the patent foramen ovale, an appropriate device including its delivery sheath can be selected. MP catheter then can be changed to the selected delivery sheath. To minimize the risk of air emboli, during this step de-bubble and flushing the catheter is crucial. The left atrial disc is deployed gradually start from the upper left pulmonary artery while keeps pulling back to patent foramen ovale and then deploy the right disc. Confirmation of adequate position using echocardiography and fluoroscopy should be performed once the device is placed before its final release.

Antibiotic regimen after the procedure is based on local hospital policy. ${ }^{12}$ Antithrombotic therapy with dual antiplatelet (DAPT) are given for 6 months after the procedure then after that single antiplatelet therapy could continue for 5 years depending on clinical features of the patient including recurrent thromboembolic \& bleeding risk.

Transthoracic Echocardiography (TTE) evaluation is done before patient discharge and the sixth week to exclude pericardial effusion and device embolization. Routine TEE performed If only there is a significant residual patent foramen ovale leakage or recurrent clinical stroke \& c-TCD is performed on the sixth month after. c-TCD evaluation is carried out every year for 5 years. Complete closure is varying from each patient's endothelialization of the device and can take up to sixth months.

The complication rate is about 1 in 14 patients undergoing transcutaneous patent foramen ovale closure, especially in older ages. ${ }^{15}$ Major adverse events could happen related to the procedure, including death, stroke, air emboli, device embolization, vascular complication, bleeding complication, Atrial Fibrillation (transient or sustained), myocardial infarction, pericardial effusion with or without tamponade. Some postprocedural major adverse events are stroke, deep vein thrombosis or pulmonary embolism if occurring within 6 months after the procedure, atrial fibrillation, atrial flutter, ventricular tachyarrhythmias, or complete heart block requiring pharmacologic therapy or cardioversion, Thrombus on the device detected, pericardial effusion, device erosion, device explantation. ${ }^{16}$

\section{Requirements for Patent Foramen Ovale Closure}

There are several requirements for operators and institutions who are allowed to performed transcutaneous patent foramen ovale Closure. These requirements are taken from the SCAI expert consensus statement on the operator and institutional requirements for patent foramen ovale closure for secondary prevention of paradoxical embolic stroke 2019 and have been modified. ${ }^{16}$

\section{Operator Requirements}

1. Should have comprehensive knowledge of atrial/ patent foramen ovale anatomy dan imaging

2. Have experience at least 50 cases of structural intervention with either minimum of 25 cases involving septal intervention or 12 cases of patent foramen ovale intervention procedures under proctor or mentor.

3. Experience with catheter-based management of potential complications, including pericardiocentesis, recognition of device malposition, and embolized device retrieval

\section{Institution Requirements}

1. Have experience at least 75 cases of structural intervention in the last 5 years.

2. Done at least 25 cases of structural intervention per year with a minimum of 10 cases involving septal intervention.

3. Have a multidisciplinary team that includes necessary staff and expertise for perioperative evaluation, performing the patent foramen ovale closure procedure, and acute and long-term postprocedural follow-up

4. Have catheterization laboratories that have been standardized by the Indonesian government authority.

5. Have good access to the cardiothoracic surgery theater to overcome the adverse event that might be happening.

6. Strongly recommended to have a patent foramen ovale Closure registry.

Before the patent foramen ovale closure procedure, 
it must be ensured that the patient and his family received information about periprocedural preparation, the intraprocedural process including the risk of adverse events, and postprocedural treatment. Education for the patient is also mandatory to increase the patient's awareness and adherence to long-term medication and treatment.

\section{Conclusion}

Closure of patent foramen ovale lesion should be done in all clinically eligible patients to improve quality of life and to prevent recurrence of paradoxical embolism. Assessment of patient eligibility can also be done more easily using available scoring sytems. With the advances in medicine, closure of patent foramen ovale can be done safely through percutaneous access. Percutaneous access can help patent foramen ovale Closure to be more widely available across centers.

\section{References}

1. Alsheikh-Ali, A. A., Thaler, D. E. \& Kent, D. M. patent foramen ovale in cryptogenic stroke: Incidental or pathogenic? Stroke (2009) doi:10.1161/STROKEAHA.109.547828.

2. Patent Foramen Ovale Closure for Stroke, Myocardial Infarction, Peripheral Embolism, Migraine, and Hypoxemia. in patent foramen ovale Closure for Stroke, Myocardial Infarction, Peripheral Embolism, Migraine, and Hypoxemia (ed. Mojadidi M Khalid, Tobis Jonathan M, M. B.) 217-219 (Elsevier Inc., 2020). doi:https://doi. org/10.1016/C2018-0-02016-2.

3. Bridges, N. D. et al. Transcatheter closure of patent foramen ovale after presumed paradoxical embolism. Circulation (1992) doi:10.1161/01.cir.86.6.1902.

4. Carroll, J. D. et al. Closure of patent foramen ovale versus medical therapy after cryptogenic stroke. N. Engl. J. Med. (2013) doi:10.1056/ NEJMoa1301440.

5. Saver, J. L. et al. Longterm outcomes of patent foramen ovale closure or medical therapy after stroke. N. Engl. J. Med. (2017) doi:10.1056/ NEJMoa1610057.

6. Mas, J. L. et al. patent foramen ovale closure or anticoagulation vs. antiplatelets after stroke. N. Engl. J. Med. (2017) doi:10.1056/NEJMoa1705915.

7. Lee, P. H. et al. Cryptogenic Stroke and HighRisk patent foramen ovale: The DEFENSE-patent foramen ovale Trial. J. Am. Coll. Cardiol. (2018) doi:10.1016/j.jacc.2018.02.046.

8. Søndergaard, L. et al. patent foramen ovale closure or antiplatelet therapy for cryptogenic stroke. N. Engl. J. Med. (2017) doi:10.1056/NEJMoa1707404.

9. Morais, L. A. et al. Rope score as a predictor of recurrent ischemic events after percutaneous patent foramen ovale closure. Int. Heart J. (2018) doi:10.1536/ihj.17-489.

10. Garg, A. et al. Recurrent Stroke Reduction with patent foramen ovaleClosureversus Medical Therapy Based on patent foramen ovale Characteristics: A Meta-Analysis of Randomized Controlled Trials. Cardiol. (2019) doi:10.1159/000500501.

11. Giblett, J. P., Abdul-Samad, O., Shapiro, L. M., Rana, B. S. \& Calvert, P. A. patent foramen ovale closure in 2019. Interv. Cardiol. Rev. (2019) doi:10.15420/icr.2018.33.2.

12. Vaduganathan, M. et al. patent foramen ovale Closure for Secondary Prevention of Cryptogenic Stroke: Updated Meta-Analysis of Randomized Clinical Trials. Am. J. Med. (2018) doi:10.1016/j. amjmed.2017.11.027.

13. Mojadidi, M. K. et al. Cryptogenic Stroke and patent foramen ovale. J. Am. Coll. Cardiol. 71, 1035-1043 (2018).

14. Baumgartner, H. et al. 2020 ESC Guidelines for the management of adult congenital heart disease. Eur. Heart J. 42, 563-645 (2021).

15. Merkler, A. E. et al. Safety outcomes after percutaneous transcatheter closure of patent foramen ovale. Stroke (2017) doi:10.1161/ STROKEAHA.117.018501.

16. Horlick, E. et al. SCAI expert consensus statement on operator and institutional requirements for patent foramen ovale closure for secondary prevention of paradoxical embolic stroke: The American Academy of Neurology affirms the value of this statement as an educational tool for neurologists. Catheter. Cardiovasc. Interv. (2019) doi:10.1002/ccd.28111. 